Pesq. Vet. Bras. 34(Supl.1):69-73, dezembro 2014 DOI: 10.1590/S0100-736X2014001300013

\title{
Osteologia do neurocrânio de Iguana iguana iguana (Squamata: Iguanidae) ${ }^{1}$
}

\author{
Fabiano C. Lima ${ }^{2 *}$, Kleber F. Pereira ${ }^{2}$, Augusto S. Abe ${ }^{3}$ e Antonio Sebben ${ }^{4}$
}

\begin{abstract}
Lima F.C., Pereira K.F., Abe A.S. \& Sebben A. 2014. [Neurocranium osteology of Iguana iguana iguana (Squamata: Iguanidae).] Osteologia do neurocrânio de Iguana iguana iguana (Squamata: Iguanidae). Pesquisa Veterinária Brasileira 34(Supl.1):69-73. Laboratório de Anatomia Humana e Comparativa, Curso de Ciências Biológicas, Universidade Federal de Goiás, Regional Jataí, Cidade Universitária, BR-364 Km 192, Setor Parque Industrial, Jataí, G0 75801-615, Brazil. E-mail: fabianocl21@hotmail.com

Skull represents the segment with conspicuous adaptations that, in lizards, may be conservative or promoted by selective pressures. The aim of assisting the morphological knowledge of reptiles, we provide a detailed description of the neurocranium of Iguana iguana iguana based on analysis of three dried adult skeletons. The skull of this species has basal characteristics in lizards without closure of cranial openings and general triangular shape. Bony structures that form the caudal base have many fusions, especially on the floor. In the caudal face the exoccipital and the opisthotic are fused and form the otooccipital, which contributes to the formation of the lateral part of the condyle. The central part is formed by the condyle supraocciopital. Fusions and skeletal structures in Iguana are similar to other lizards. There are no autopomorphies in the neurocranium for this species.
\end{abstract}

INDEX TERMS: Iguana iguana iguana, green iguana, reptiles, neurocranium, skull, skeleton.

RESUMO.- O crânio representa o segmento com conspícuas adaptações que, nos lagartos, podem ser conservativas ou impulsionadas por pressões seletivas. Objetivando subsidiar o conhecimento morfológico dos répteis, fornecemos uma descrição detalhada dos ossos que formam o neurocrânio de Iguana iguana iguana com base na análise de três esqueletos secos de espécimes adultos. 0 crânio da referia espécie possui características basais entre os lagartos sem o fechamento das aberturas cranianas e formato geral triangular. As estruturas ósseas que formam a base craniana apresentam muitas fusões, principalmente no assoalho. $\mathrm{Na}$ face caudal o exoccipital e o opistótico estão fundidos

\footnotetext{
${ }^{1}$ Recebido em 6 de outubro de 2014.

Aceito para publicação em 30 de dezembro de 2014.

${ }^{2}$ Laboratório de Anatomia Humana e Comparativa, Curso de Ciências Biológicas, Universidade Federal de Goiás, Regional Jataí, Cidade Universitária, BR-364 Km 195, Setor Parque Industrial, Jataí, GO 75801-615, Brasil.*Autor para correspondência: fabianocl21@hotmail.com

${ }^{3}$ Departamento de Zoologia, Instituto de Biociências de Rio Claro, Universidade Estadual Paulista Júlio de Mesquita Filho. Avenida 24A, 1515, Cx. Postal 199, Bela Vista, Rio Claro, SP 13506-900, Brasil.

${ }^{4}$ Laboratório de Anatomia Comparativa dos Vertebrados, Departamento de Ciências Fisiológicas, Universidade de Brasília, Campus Darcy Ribeiro, Instituto de Ciências Biológicas, Bloco E, térreo, guichê da Pós-Graduação em Biologia Animal, Brasília, DF 70910-900, Brasil.
}

e formam o otoccipital, que contribui para a formação dos terços laterais do côndilo occipital. A parte central do côndilo é formada pelo supraoccipital. Fusões e estruturas esqueléticas presentes em Iguana são similares aos demais lagartos. Não foram descritas autapomorfias no neurocrânio para esta espécie.

TERMOS DE INDEXAÇÃ̃: Iguana iguana iguana, iguana verde, répteis, crânio, esqueleto.

\section{INTRODUÇÃO}

Empregado como ferramenta para desvendar aspectos da biologia evolutiva e na reconstrução de filogenias (Shubin \& Alberch 1986, Mabee et al. 2000, Gilbert et al. 2001), o conhecimento morfológico auxilia também na compreensão das tendências funcionais e das preferências ambientais em diferentes estágios de desenvolvimento. 0 esqueleto é suficientemente conservativo, a ponto do seu padrão geral mostrar as amplas linhas filogenéticas dos vertebrados, desempenhando fundamental papel funcional, apresentando plasticidade para responder aos hábitos particulares dos vários animais (Hildebrand \& Goslow 2006).

Dentre os elementos esqueléticos, o crânio dos vertebrados corresponde ao segmento corporal com as maiores adaptações e responde de maneira satisfatória as necessi- 
dades destes animais. Desempenha funções importantes para proteção, estabilidade e acuidade de todos os sistemas neuro-sensoriais da cabeça e dos aparelhos de alimentação e respiração (Kearney et al. 2005, Herrel et al. 2007). Cada grupo de animais possui características distintas em sua anatomia craniana o que, dentre outras aplicações, são utilizadas de ampla forma para a descrição de relações filogenéticas (Rieppel 1984, Benton 1985, Herrel et al. 2007, Conrad \& Norell 2010, Jerez 2012). Nos lagartos, sua anatomia pode ser conservativa em para algumas características sendo impulsionada pelas demandas de construção impostas por pressões seletivas. Assim, este segmento do esqueleto é ideal para explorar como as diferentes pressões seletivas e restrições tendem a agir para moldar a evolução de um sistema integrado complexo (Herrel et al. 2007).

Popularmente como iguana verde, Iguana iguana iguana (Linnaeus, 1758) se distribui pelas Américas e no Brasil ocorre nos biomas Amazônia, Caatinga, Pantanal (Campos 2004, Swanson 2004, Campos \& Desbiez 2013). Possuem hábitos arborícolas (Lazel 1973, Swanson 2004) e cavam os ninhos no solo (Rodda 1990). Pertencem a família Iguanidae, cujas características diagnósticas são, fusão da premaxila, presença de tuberosidade prefrontal, processo ventromedial do pterigóide, quilhas presentes na vértebra cervical e planos de autotomia caudal presentes posteriormente aos processos transversais (Conrad 2004).

Embora existam algumas descrições da morfologia craniana de lagartos do grupo Iguania (Abdala et al. 1997, Torres-Carvajal 2003, Silva \& Verrastro 2007, Banzato et al. 2012), os relatos acerca da anatomia pormenorizada de $I$. iguana iguana são genéricos ou relatam pontualmente alguns aspectos (Conrad \& Norell 2010, Banzato et al. 2012). Objetivando contribuir com o conhecimento morfológico dos lagartos, fornecemos aqui uma descrição detalhada do neurocrânio de I. iguana iguana.

\section{MATERIAL E MÉTODOS}

Três espécimes adultos de I. iguana iguana (dois machos e uma fêmea) que morreram por causas naturais no Jacarezário da UNESP de Rio Claro foram preparados seguindo métodos osteológicos usuais. Todo tecido mole (tegumento, músculos e vísceras) foi removido com auxílio de bisturi, tesoura e faca deixando o esqueleto exposto e o mais limpo possível. Alguns segmentos foram desarticulados (membros, coluna vertebral, cauda e crânio) e levados à cocção por uma hora, retirados o excesso de tecido mole, e banhados com água quente por mais 30 minutos. Estes foram colocados em solução de peróxido de hidrogênio (3\%), identificados, fotografados e documentados.

A colônia de iguanas mantidas na Unesp-Rio Claro/SP é devidamente licenciada pelo ICMBIO-IBAMA no.973766. A investigação está amparada pelo parecer do comitê de ética da Universidade Federal de Uberlândia sob protocolo CEUA 070/2012. A utilização do material cadavérico animal é respaldado pela Instrução Normativa número 154/2007 do IBAMA e pela legislação vigente (Lei número 11.794/2008 que regulamenta Pesquisas com Animais no Brasil).

\section{RESULTADOS}

Basioccipital (Basioccipitale). Está posicionado entre as capsulas ópticas e forma a parede caudal da caixa craniana e a porção media do côndilo occipital (condylus occipitalis). Articula-se amplamente com o parabasisfenóide rostralmente. Articula-se com o processo inferior do pró-ótico (processus inferioris) rostrolateralmente e com a crista tuberal do otoccipital (crista tuberalis) laterocaudalmente (Fig.1B, C).

Supraoccipital (Supraoccipitale). Está orientado rostrodorsalmente. Apresenta forma similar a uma sela que repousa caudoventralmente ao parietal e forma a porção caudal do teto do crânio. Sua porção medial forma a borda dorsal do forame magno (foramen magnum). A margem ventrolateral articula-se com a margem dorsolateral do processo alar do pró-ótico (processus alaris) rostralmente e com a margem posterocaudal do corpo do otoccipital caudalmente. As faces ventrocaudal e dorsocaudal possuem uma depressão, um recesso côncavo orientado anteromedialmente que constitui a metade anterior da bula auditória (auditory bulla), a metade anterior é formada pelo otoccipital. Sua porção mais caudal possui duas depressões côncavas lateralmente orientadas. Na porção mediana desta face, uma crista protuberante e delgada separa as referidas depressões. A superfície dorsal contata o parietal e exibe, próximo a linha mediana, dois pequenos tubérculos lateralmente dispostos a crista mediana do supraoccipital (Fig.1C).

Parabasisfenóide (Parabasisphenoidale). A porção dérmica deste elemento é fundida com o basisfenóide e, portanto, indistinguível, exceto por um longo e delgado processo cultriforme rostromedial (processus cultriforme). Assim, esses ossos são descritos genericamente como um único elemento assim denominado. Ele forma o assoalho do crânio e se articula com o basioccipital caudalmente e com o pró-ótico dorsalmente. Suas bordas ventrolateral e rostrodorsal são formadas pela crista selar transversa (crista sellaris). Ele possui três pares de processos em adição ao processo cultriforme. Dois delgados, planos e laterocaudais processos que sobrepõem o basioccipital rostrolateralmente e se estendem na face rostrodorsal até o tubérculo esfenoccipital (tuberculum sphenoccipitale). Entre as extremidades destes processos, a superfície ventral apresenta um recesso. Rostralmente dois processos basipterigóideos (processus basipterigoideus), orientados ventrolateralmente, estão presentes e expandem esta extremidade. Esta porção articula-se com o pterigóide. Dorsal a cada processo basipterigóide existe um pequeno processo alar dorsolateral (processus alaris). As extremidades deste processo e da porção anterior do processo anteroventral do pró-ótico estão aderidos à pila antótica (pilae antotica). Lateralmente é perfurado, na base de cada processo posterolateral, por um canal carótico (canalis caroticus), que percorre a porção ventrolateral na superfície anterior. 0 delgado e alongado processo cultriforme repousa sobre a trabécula comum (trabecula communis) e se estende rostrodorsalmente até o ponto correspondente ao limite posterior da maxila, dorsalmente ao espaço piriforme (Fig.1).

Otoccipital (Otoccipitale). Formando pela fusão do exoccipital e opistótico. Estes elementos serão então descritos como únicos. Este forma a parede posterior do crânio e contata o pró-ótico rostrolateralmente, o supraoccipital 
dorsomedialmente e o basioccipital ventromedialmente. Forma a margem lateral do forame magno e a porção late-

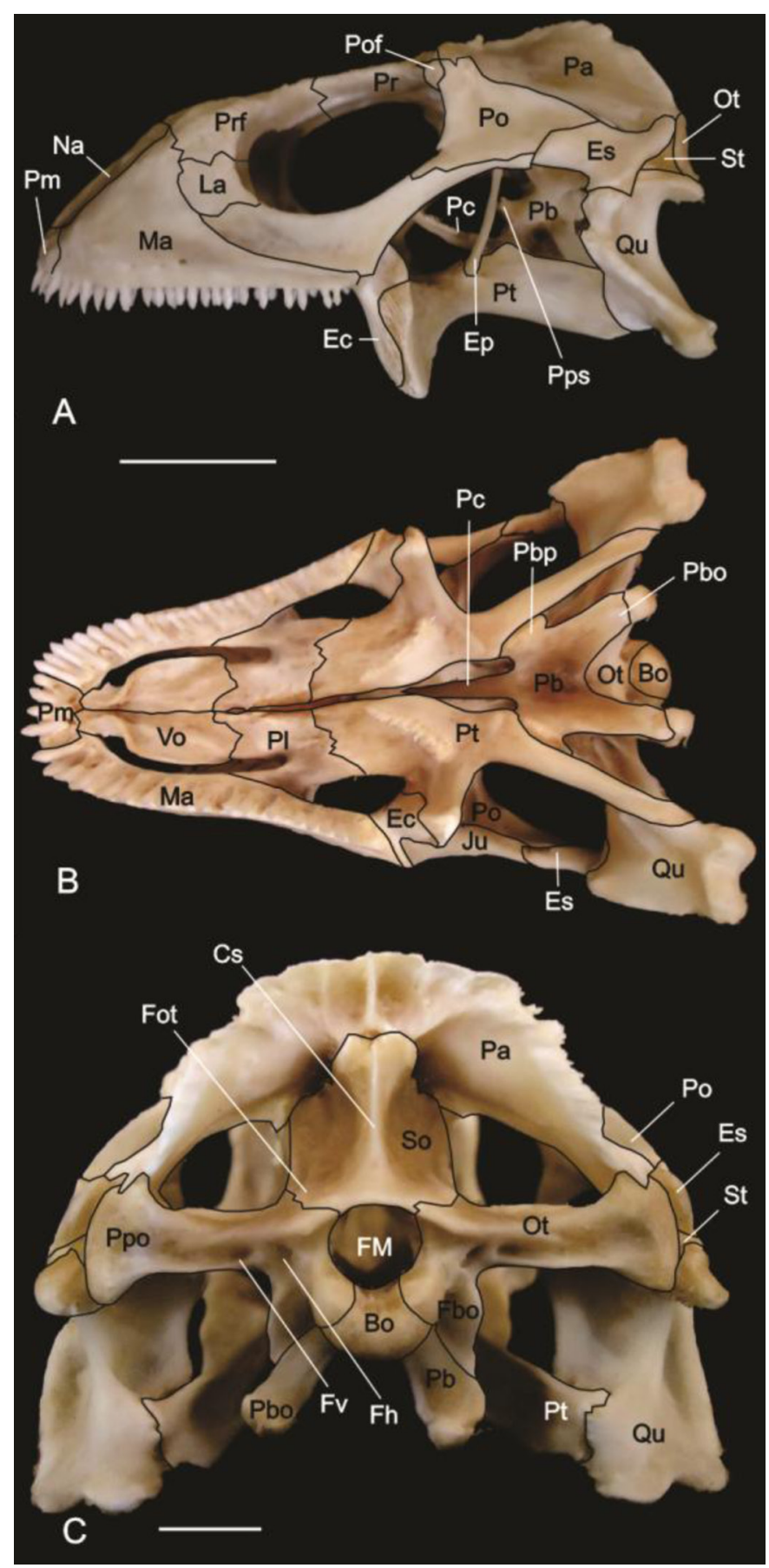

Fig.1. (A) Crânio de Iguana iguana iguana em vista lateral, (B) vista palatal e (C) vista caudal. Basioccipital (Bo), crista sagital (Cs), ectopterigóide (Ec), epipterigóide (Ep), esquamosal (Es), faceta basioccipital (Fbo), forame hipoglosso (Fh), forame magno (FM), faceta otoccipital (Fot), forame vagal (Fv), jugal (Ju), lacrimal (La), maxila (Ma), nasal (Na), otoccipital (Ot), parietal $(\mathrm{Pa})$, parabasisfenóide $(\mathrm{Pb})$, processo basioccipital $(\mathrm{Pbo})$, processo basipterigóide $(\mathrm{Pbp})$, processo cultriforme $(\mathrm{Pc})$, pré-frontal (Pfr), palatino (Pl), pré-maxila (Pm), pós-orbital (Po), pós-frontal (Pof), processo paroccipital (Ppo), processo paresfenóide (Pps), frontal (Pr), pterigoide (Pt), quadrado (Ou), supraoccipital (So), supratemporal (St), vômer (Vo). Barra $=2 \mathrm{~cm}$. ral do côndilo occipital. Possuem um processo paroccipital (processus par occipitalis). A superfície interna deste possui uma depressão côncava em forma de recesso que compreende na metade posterior da bula auditória. Os forames hipoglosso e vagal estão presentes na porção caudal. 0 processo paroccipital estende-se laterocaudalmente como um par de barras robustas e quadrangulares. Sua porção lateral apresenta uma pequena dilatação similar a um tubérculo que contata o quadrado, o parietal e o supratemporal. A extremidade lateral de cada processo paroccipital é sobreposta pela extremidade posterior do supratemporal rostralmente, o processo supratemporal do parietal (processus supratemporalis) dorsalmente e a ventralmente. 0 esquamosal também contata discretamente este. Anterior ao processo paroccipital, cada otoccipital possui um recesso jugular (recessos jugularis). Caudalmente exibe uma crista tuberal (crista tuberalis), que possui lateralmente um forame hipoglosso ventral (canalis nervus hipoglossi ventralis). Esta porção do otoccipital ainda possui três forames hiploglossos juntamente dispostos com o forame vagal (Fig.1).

Orbitoesfenóide (Orbitosphenoidei). Correspondem em um par de elementos ossificados na parte anterior do neurocrânio. São alongados, côncavos caudalmente, cranialmente ao parabasisfenóide e cartilagem intercalar (cartilago intercalaris) medialmente dispostos aos epipterigóides. Limitam a margem lateral da fenestra óptica (fenestra optica).

Pró-ótico (Prooticum). Posicionados na parede rostrolateral do crânio possui, cada um, três processos, o processo alar (processus alaris), o rostroventral (processus rostroventralis) e o dorsocaudal (processus dorsocaudalis). 0 processo alar é pequeno e rostrodorsalmente orientado com a margem lateral do supraoccipital, caudalmente. A margem rostral do processo alar forma a crista alar (crista alaris). 0 processo rostroventral articula-se com o parabasisfenóide rostralmente e com o basioccipital ventralmente. Este possui ventralmente uma ampla cavidade perfurada pelo forame facial (foramen facialis). A margem lateral deste processo forma a metade anterior da crista pró-ótica. 0 processo dorsocaudal sobrepõe a superfície rostral do processo paraoccipital do otoccipital e a margem medial forma a margem anterior da fenestra oval. Este recesso forma o assoalho e a parede medial da cavidade timpânica.

\section{DISCUSSÃO}

De maneira geral o crânio mantém as características de lagartos ancestrais, sem o fechamento das aberturas cranianas (George \& Smallwood 1992). George \& Smallwood (1992) e Banzato et al. (2012) relatam que o crânio de Iguania é grosseiramente triangular na vista dorsal e com uma pequena região pré-orbital, mesmas características encontradas em I. iguana iguana. Dentre as espécies de lagartos, aquelas que apresentam tamanho reduzido possuem crânios largos e fundidos (Rieppel 1984, Müller 2002). Em I. iguana iguana o crânio pode ser considerado grande em relação ao grupo de lagartos no geral, mas esta é uma característica relacionada ao porte do animal. As estruturas ósseas estão bem delimitadas e não existem processos aparentes de fusão, exceto na parte caudal e assoalho craniano.

Posicionados na parte caudal, os ossos que limitam a 
caixa craniana são o basioccipital, supraoccipital, exoccipital, opstótico (ou otoccipital, quando estão fundidos), parabasisfenóide, proótico, epiótico e orbitoesfenóide. Supraoccipital e basioccipital são ossos ímpares, e formam parte do teto e assoalho craniano, respectivamente. Este último contribui para a parte mediana do côndilo. Em Anolis, Scincus e Lygosma não é possível averiguar a sutura entre o basioccipital e o exoccipital, sendo o côndilo formado provavelmente apenas pelo basioccipital. Tal sutura é perfeitamente diferenciada em I. iguana iguana, evidenciando a contribuição de elementos ósseos distintos na formação do côndilo, padrão averiguado nos demais lagartos (Romer 1956, Torres-Carvajal 2003, Khosravani et al. 2011) Lateralmente nesta região está posionado o par de proóticos que, nos lagartos adultos, não apresentam divisão conspícua entre os demais ossos do neurocrânio (Simões-Lopes e Krause, 1988) e limitam lataralmente o forame magno.

Vários são os relatos de fusão de estruturas do neurocrânio (pro-ótico, opistótico e exoccipital principalmente). Todos estes elementos possuem centros de ossificação distintos e são, normalmente, diferenciáveis nos jovens (Stephenson \& Stephenson 1956). Sua fusão pode estar associada ao grau de ossificação do crânio nestes animais (Jolie 1960).

O otoccipital é par e lateralmente disposto na face caudal do crânio. Em L. occipitalis ele é formado pela união do exoccipital e opistótico, sendo este último diferenciado em algumas espécies (Oelrich 1956). Estes elementos estão fundidos em I. iguana iguana, tal como em Stenocercus guentheri (Torres-Carvajal 2003), Varanus prisca (Head et al. 2009), Eremias persica, Measalina watsonana (Khosravani et al. 2011), Shinisaurus crocodilurus (Bever et al. 2005), Naultinus (Stephenson \& Stephenson 1956), Ctenosauria pectinata e Sator grandaevus (Oelrich 1956, Etheridge 1962) dentre outros. A fusão acontecem ainda durante o desenvolvimento pré-natal em muitos lagartos (Maisano 2001) mas pode eventualmente ocorrer em jovens tal como relatado para $S$. crocodilurus (Bever et al. 2005). Este elemento forma o terço lateral do côndilo e a borda lateral do forame magno, delimitando a parede posterolateral do crânio. 0 par de processos paroccipitais são amplos e lateralmente dispostos contatando os osso quadrado, parietal e esquamosal (Head et al. 2009). 0 opstótico, quando diferenciado, é muito reduzido (Stephenson \& Stephenson 1956), embora nenhuma das descrições, inclusive para I. iguana iguana tal morfologia tenha sido observada.

Contribuindo na delimitação da margem rostral do processo paroccipital, o pró-otico forma a parede anterolateral do neurocrânio nos répteis em geral (Stephenson \& Stephenson 1956, Torres-Carvajal 2003, Bever et al. 2005, Bell et al. 2009, Head et al. 2009, Khosravani et al. 2011). Na transição com a região orbital, o orbitoesfenóide (lateroesfenóide, Romer 1956) é uma ossificação da parte dorsal da pila metótica, localizada na parte anterior da caixa craniana que contribui para a formação da parede posterior da órbita (Bellairs \& Kamal 1981, Torres-Carvajal 2003, Bell et al. 2009). Nos répteis, tal como em I. iguana iguana, trata-se de um pequeno par de ossos curvados que se formam próximo a linha mediana, anteriormente ao parabasisfenóide e medialmente aos epipterigóides. Bever et al. (2005) relatam sua presença em $S$. crocodilurus, embora Conrad (2004) e outros autores não tenham descrito o orbitoesfenóide na referida espécie. Em Xenosaurus sua presença é variável, e a ausência é relatada em Gekkota, Heloderma e Anniella (Jolie1960), representando provavelmente variações intraespecíficas ou mesmo problemas durante o preparo do material. Tarazona et al. (2008) relatam que em Bachia bicolor tratam-se de um largo e laminar par de ossos posicionados próximo a linha mediana, fechando anteriormente o neurocânio. Tal característica é similar as espécies do grupo Amphisbaenia e diferente dos demais répteis, onde estes normalmente não contatam outros ossos do crânio.

0 parabasisfenóide foi descrito em diversos lagartos (Head et al. 2003) e com a mesma topografia encontrada em Iguana, anterolateral na base do neurocânio. Abriga a cela turca, bem como os forames para artérias cerebrais e o nervo craniano VI. As relações filogenéticas e a anatomia deste elemento apresentam uma extensa discussão (Jolie 1960, Bellairs \& Kamal 1981). 0 padrão atribuído aos répteis é a fusão do paresfenóide e basisfenóide formando este elemento ímpar. Eremias persica, Measalina watsonana (Khosravani et al. 2011), Varanus (Head et al. 2003), S. crocodilurus (Bever et al. 2005), Moloch horridus (Bell et al. 2009) e I. iguana iguana apresentam os elementos paresfenóide e basisfenóide fundidos formando um único parabasisfenóide (Rieppel 1993, Torres-Carvajal 2003). Em Chamaeleo o paresfenóide é distinto, embora seja pouco desenvolvido. 0 processo cultriforme (rostro parabasisfenóide) está usualmente fundido a placa basal, podendo ser laminar ou como em Iguana, afilado e projetado rostralmente, alcançando a metade da extensão da órbita. Bever et al. (2005) relatam que em $S$. crocodilurus existe uma fontanela basicranial em alguns espécimes que apresentam uma área não ossificada na sutura basioccipital. Possivelmente seja uma heterocronia, haja vista não ser uma exclusividade da espécie e estar relacionado a idade dos espécimes. Nos exemplares de $I$. iguana iguana que avaliamos, todos adultos, a sutura estava visivelmente bem ossificada.

\section{CONCLUSÕES}

O Neurocrânio de I. iguana iguana possui todos os elementos ósseos descritos nos demais lagartos. Não foram descritas características particulares para a espécie na região do neurocrânio.

A fusão entre o opstótico e o exoccipital forma o otoccipital, e entre o paresfenóide e basisfenóide origina o parabasisfenóide tal como na maioria dos lagartos.

A parede anterior do crânio é aberta constando apenas o par de barras curvas do orbitoesfenóide que não se articulam com os demais ossos do crânio.

A morfologia geral desta estrutura é conservativa dentre os lagartos.

Agradecimentos.- Às biólogas Marina Sartori e Lucélia Vieira e ao professor André Quagliatto pelo empenho na aquisição do material. 


\section{REFERÊNCIAS}

Abdala F., Lobo F. \& Scrocchi G. 1997. Patterns of ossification in the skeleton of Liolaemus quilmes (Iguania: Tropiduridae). Amp. Rep. 18:75-83.

Banzato T., Selleri P., Veladiano I.A., Martin A., Zanetti E. \& Zotti A. 2012. Comparative evaluation of the cadaveric, radiographic and computed tomographic anatomy of the heads of green iguana (Iguana iguana), common tegu (Tupinambis merianae) and bearded dragon (Pogona vitticeps). BMC Vet. Res. 8:53.

Bell C.J., Mead J.I. \& Swift S.L. 2009. Cranial osteology of Moloch horridus (Reptilia: Squamata: Agamidae). Rec. West. Aust. Mus. 25:201-237.

Bellairs A.d'A. \& Kamal A.M. 1981. The chondrocranium and the development of the skull in recent reptiles, p.1-283. In: Gans C. \& Parsons T.S. (Eds), Biology of the Reptilia: morphology. Academic Press, New York.

Benton M.J. 1985. Classification and phylogeny of diapsid reptiles. Zool. J. Lin. Soc. 84:97-164.

Bever G.S., Bell C.J. \& Maisano J.A. 2005. The ossified braincase and cephalic osteoderms of Shinisaurus crocodilurus (Squamata, Shinisauridae). Paleontol. Elect. 8:1-36.

Campos Z. \& Desbiez A.L.J. 2013. Structure of size and reproduction of green iguanas (Iguana iguana) in the Brazilian Pantanal. IRCF Reptiles and Amphibians 20: 53-56.

Campos Z. 2004. Iguana iguana (Sinimbu). Reproduction. Herpetol. Rev. 25:169.

Conrad J.L. \& Norell M.A. 2010. Cranial autapomorphies in two species of Iguana (Iguanidae: Squamata). J. Herpetol. 44:307-312.

Conrad J.L. 2004. Skull, mandible, and hyoid of Shinisaurus crocodilurus Ahl (Squamata, Anguimorpha). Zool. J. Lin. Soc. 141:399- 434.

Etheridge R. 1962. Skeletal variation in the Iguanidae lizard Sator grandaevus. Copeia 1962:613-619.

George T.F. \& Smallwood J.E. 1992. Anatomic atlas for computed tomography in the mesaticephalic dog: head and neck. Vet. Radiol. Ultrasound $33: 217-240$.

Gilbert S.F., Loredo G.A., Brukman A. \& Burke A.C. 2001. Morphogenesis of the turtle shell: the development of novel structure in tetrapod evolution. Evol. Dev. 3:47-58.

Herrel A., Schaerlaeken C., Meyers J.J., Metzger K.A. \& Ross C.F. 2007. The evolution of cranial design and performance in squamates: Consequences of skull-bone reduction on feeding behavior. Integr. Comp. Biol. 47:107-117.

Hildebrand M. \& Goslow G.E. 2006. Análise da estrutura dos vertebrados. Atheneu, São Paulo.

Jerez A. 2012. Caracteristicas estruturales del esqueleto en Mabuya sp. (Squamata: Scincidae): una comparatión con escíncidos africanos. Actualidades Biológicas 34:207-223.

Jolie M.T. 1960. The head skeleton of the lizard. Acta Zool. 41:1-64.
Kearney M., Maisano J.A. \& Rowe T. 2005. Cranial anatomy of the extinct Amphisbeanian Rhineura hatcherii (Quamata, Amphisbaenia) Based on High-Resolution X-Ray Computed Tomography. J. Morphol. 264:1-33.

Khosravani A., Rastegar-Pouyani N. \& Oraie H. 2011. Comparative skull osteology of the lacertid lizards Eremias persica and Mesalina watsonana (Sauria: Lacertidae). Iran. J. Ani. Biosyst. 7:99-117.

Lazell J.D. 1973. The lizard genus Iguana in the Lesser Antilles. Bul. Mus. Comp. Zool. 145:1-28.

Mabee P.M., Otmstead K.L. \& Cubbage C.C. 2000. An experimental study of intraspecific variation, developmental, timing, and heterochrony in fishes. Evol. 54:2091-2106.

Maisano J.A. 2001. A survey of state of ossification in neonatal squamates. Herpetol. Monogr. 15:135-157.

Müller J. 2002. Skull osteology of Parvilacerta parva, a small-sized lacertid lizard from Asia Minor. J. Morphol. 253:43-50.

Oelrich T.M. 1956. The anatomy of the head of Ctenosaura pectinata (Iguanidae). University of Michigan, Michigan.

Rieppel 0. 1984. Miniaturization of the lizard skull: its functional and evolutionary implications. Symp. Zool. Soc. Lond. 52:503-520.

Rieppel 0.1993. Patterns of diversity in the reptilian skull, p. 344-390. In: Hanken J. \& Hall B.K. (Eds), The Skull: patterns of structural and systematic diversity. University of Chicago Press, Chicago.

Rodda G.H. 1990. Highway madness revisited: Roadkilled Iguana iguana in the llanos of Venezuela. J. Herpetol. 24:209-211.

Romer A.S. 1956. Osteology of the Reptiles. University of Chicago Press, Chicago.

Shubin N.H. \& Alberch P.A. 1986. Morphogenetic approach to the origin and basic organization of the tetrapod limb. Evol. Biol. 20:318-390.

Silva C.M. \& Verrastro L. 2007. Descrição do esqueleto axial de Liolaemus arambarensis Verrastro, Veronese, Bujes \& Dias Filho (Iguania, Liolaemidae): regiões pré-sacral e sacral. Revta Bras. Zool. 24:1-11.

Simões-Lopes P.C.A. \& Krause L. 1988. Osteologia do sincrânio de Liolaemus occipitalis Boulenger, 1885 (Sauria, Iguanidae). Reta. Bras. Zool. 5(4):491-508.

Stephenson N.B. \& Stephenson M. 1956. The osteology of New Zealand geckos and its bearing on their morphological status. Trans. R. Soc. New Zealand 84:341-385.

Swanson P.L. 2004. The iguana: Iguana iguana iguana. Iguana 11:179-182.

Tarazona O.A., Fabrezi M. \& Ramírez-Pinilla M.P. 2008. Cranial morphology of Bachia bicolor (Squamata: Gymnophthalmidae) and its postnatal development. Zool. J. Lin. Soc. 152:775-792.

Torres-Carvajal O. 2003. Cranial Osteology of the Andean Lizard Stenocercus guentheri (Squamata: Tropiduridae) and Its Postembryonic Development. J. Morphol. 255:94-113. 